\title{
A dynamical study of the Gefion asteroid family
}

\author{
S. Aljbaae ${ }^{1}$, J. Souchay ${ }^{2}$, A. F. B. A. Prado ${ }^{1}$, and T. G. G. Chanut ${ }^{1}$ \\ ${ }^{1}$ Division of Space Mechanics and Control, INPE, C.P. 515, 12227-310 São José dos Campos, SP, Brazil \\ e-mail: safwan.aljbaae@pq.cnpq.br \\ 2 SYRTE, Observatoire de Paris, PSL Research University, CNRS, Sorbonne Universités, UPMC Université Paris 06, LNE, \\ 61 avenue de l'Observatoire, 75014 Paris, France
}

Received 19 October 2018 / Accepted 28 November 2018

\begin{abstract}
The Gefion asteroid family is a group of S-type asteroids located between the 8J:-3A and 5J:-2A mean-motion resonances. The 5J:-2A resonance seems to be responsible for the absence of the right side of the $\mathrm{V}$-shape of this family. We aim in this work to present a detailed study on the Gefion family, motivated by the incompatibility found in previous family age estimations and the fact that this family could be seen as one of the most probable sources of L-chondrite meteorites. After eliminating all possible taxonomical and dynamical interlopers, we use a Monte Carlo method to analyze the semi-major axis evolution of several fictitious families under the influence of the Yarkovsky and Yarkovsky-O'Keefe-Radzievsky-Paddack (YORP) effects. We also perform simulations using symplectic integrators to account for the Yarkovsky effect (diurnal and seasonal versions) and the stochastic YORP effect. We make use of the distribution of the component of the ejection velocity field $\left(v_{W}\right)$ perpendicular to the orbital plane and the time dependence of the asymmetry of the distribution of the target function of a fictitious family generated with ejection velocity parameter $20_{-15}^{+55} \mathrm{~m} \mathrm{~s}^{-1}$ to obtain an age estimate of $1030_{-67}^{+19} \mathrm{Myr}$. We find that $6.5 \%$ of asteroids from the Gefion family can reach orbits similar to particles in the current near-Earth objects space; $73 \%$ of them are among the Amors asteroids, and the remaining ones are among the Apollos. We only found $0.5 \%$ from the Gefion family reaching the Mars-crossing space.
\end{abstract}

Key words. minor planets, asteroids: general - celestial mechanics - gravitation

\section{Introduction}

The family of (1272) Gefion is a group of S-type asteroids located in the intermediate asteroid belt close to (1) Ceres, between the $8 \mathrm{~J}:-3 \mathrm{~A}$ and $5 \mathrm{~J}:-2 \mathrm{~A}$ mean-motion resonances (MMRs) with Jupiter. This makes the family of Gefion particularly interesting for predictions of the exogenic material that can be found on Ceres (Le Corre et al. 2015). The Gefion family is crossed by MMRs of Jupiter (18J:-7A, 23J:-9A) and Mars (7M:-17A). The very strong 5J:-2A resonance at 2.82362 au caused the loss of asteroids at the high proper semi-major axis $(a)$ of the family (Spoto et al. 2015). The Gefion family was selected as one of the possible sources of L-chondrite meteorites due to its location near the $5 \mathrm{~J}$ :-2A MMR, which is capable of transporting meteorites to the Earth (Nesvorný et al. 2009). Investigating the changes in the semimajor axis produced by close encounters with massive asteroids on Gefion family members, Carruba et al. (2003) found that the family is less than 850 Myr old. Using Eq. (1) in Nesvorný et al. (2015), the age estimated by Carruba \& Nesvorný (2016) was $980 \pm 290$ Myr. Spoto et al. (2015) estimated the age of this family to be $1103 \pm 386 \mathrm{Myr}$, considering that one of the two sides of its V-shape is nonidentifiable. This estimation is in good agreement with the results of Nesvorný et al. (2005), who gave a value of $1.2 \pm 0.4$ Gyr. Nesvorný et al. (2009) found a discordant age of $485_{-10}^{-40}$ Myr. We note that the latter authors applied a method that allows for the ages of young asteroid families to be estimated, developed in Vokrouhlický et al. (2006b). They performed simulations in which fictitious families were generated from the assumed breakup site $\left(a_{\text {break }}=2.979\right.$ au, outside the background of our family) for different speeds following a Maxwellian distribution with the specified mean ejection speed (see Sect. 4). This method is sensitive to the semimajor axis location of the breakup and the thermal parameters necessary for modeling Yarkovsky forces. A thermal conductivity of $K=0.01 \mathrm{~W} \mathrm{~m}^{-1} \mathrm{~K}^{-1}$ was used in Nesvorný et al. (2009) that is ten times bigger than the typical thermal parameters of S-type bodies listed in Brož et al. (2013); this gives larger Yarkovsky mobility and younger ages. The differences of these age estimations motivated us to start a detailed analysis in the region where we try to refine the initial age estimation and check the contribution of the region to the near-Earth objects (NEOs) population.

According to Nesvorný et al. (2015), the Gefion dynamical group (family identification number, FIN $=516$ ) consists of 2547 members, identified by the hierarchical clustering method (HCM, Zappala et al. 1994; Bendjoya \& Zappalà 2002) with a cutoff distance of $50 \mathrm{~m} \mathrm{~s}^{-1}$. This corresponds to an interval in $a$ from 2.70463 to $2.81587 \mathrm{au}$, in $e$ from 0.08333 to 0.15568 , and in $\sin i$ from 0.14442 to 0.16998 . Ceres, with a proper orbital semimajor axis of 2.767 au was thought to be a member of this family; indeed, it shares similar proper orbital elements but with spectral properties different from other members (Cellino et al. 2002). Milani et al. (2014) identified 2313 asteroids (2042 are numbered whereas 271 are in multi-opposition) in this family, considering $90 \mathrm{~m} \mathrm{~s}^{-1}$ as the critical quasi-random level (QRL). The authors showed that (93) Minerva is the lowest-numbered member and therefore opted to name the cluster after Minerva. The proper orbital elements of the Minerva family vary from 2.71968 to 2.81562 au in $a$, from 0.11545 to 0.15481 in $e$, and from 0.14583 to 0.16932 in $\sin i$. In Fig. 1, we present the orbital location of the Gefion HCM family members, and of the Minerva family ones with respect to (1272) Gefion itself. We can observe 

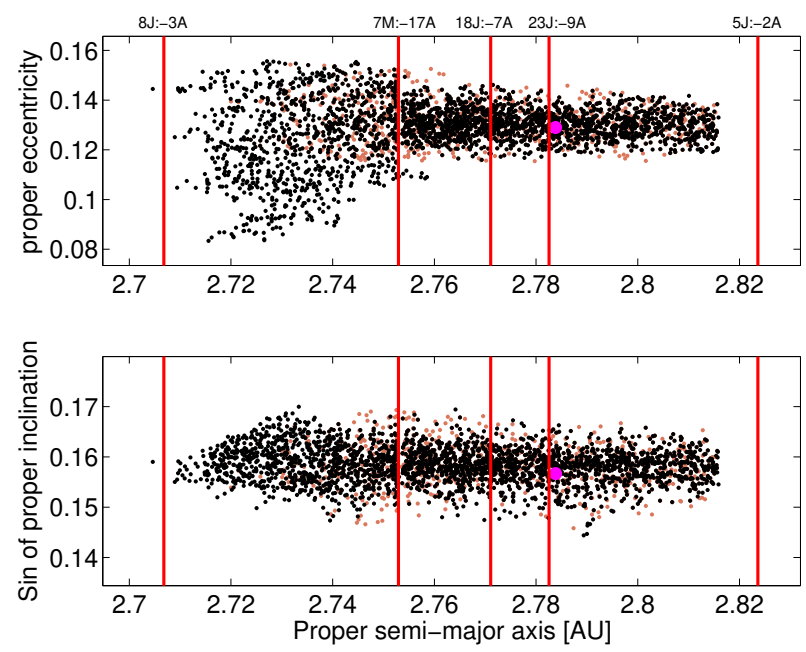

Fig. 1. Top panel: projection in the $(a, e)$ domain of 2547 members of the Gefion family (black) and of 2313 members of the Minerva family (orange). Bottom panel: projection in the $(a, \sin i)$ domain of the same asteroids. Vertical red lines display the strongest main MMR. In both panels, the point in magenta corresponds to the asteroid (1272) Gefion.

a relatively large spread in proper eccentricity of Gefion members, probably due to the MMR resonance with Mars 7:-17 at $a<2.75$ au. However, in Spoto et al. (2015), (93) Minerva was identified as an interloper due to its albedo (0.073), which is different from the mean albedo of the family. Despite the presence of numbered and multi-opposition objects in the identification of the Minerva group, this group is $\sim 10 \%$ smaller than the Gefion dynamical group. In this work, we use the data from Nesvorný et al. (2015) to investigate the orbital evolution of the members of the Gefion family. First, in Sect. 2, we revise the current knowledge on the taxonomy and physical properties of asteroids in the family region. Then, in Sect. 3, we investigate the local dynamics in this region. In Sect. 4 we use the method of Yarkovsky isolines and Yarko-YORP Monte Carlo approaches to obtain estimates of the family age. Finally, in Sect. 5, we investigate the dynamical evolution of the Gefion family, by testing two dynamical models, including or not the stochastic YORP effect. Finally, we present our conclusions in Sect. 6.

\section{Physical and taxonomic properties in the Gefion region}

There are 5523 objects found in the background of Gefion family, which correspond to objects from the AstDyS ${ }^{1}$ database within the minimum and maximum values \pm 0.01 of the proper $a, e$, and $\sin i$ with respect to the members of the family (see Fig. 2).

Using the feature-based taxonomy of Bus \& Binzel (2002a,b), the Small Main-belt Asteroid Spectroscopic Survey (SMASS $\mathrm{II}^{2}$ ) identified 75 asteroids in the background of the Gefion family (1:A, 1:B, 5:C, 2:Cb, 5:Ch, 42:S, 3:S1, 5:Sq, 4:Sr, $1: \mathrm{X}, 1: \mathrm{Xc}, 1: \mathrm{Xe}, 4: \mathrm{Xk})$. Among them, 35 are in the Gefion HCM group (1:C, 29:S, 2:S1, 1:Sq, 2:Sr). Moreover, the fourth Release of the Sloan Digital Sky Survey Moving Object Catalog (SDSS$\mathrm{MOC}^{3}{ }^{3}$, Ivezić et al. 2001) listed 606 objects in the background. Of these, 332 are in the HCM group. From the 606 objects of

\footnotetext{
1 ftp://hamilton.dm.unipi . it/astdys/ accessed on June 2017

2 http://smass.mit.edu/smass.html

3 ftp://www.astro.washington.edu/users/ivezic/sdssmoc/ sdssmoc.html
}
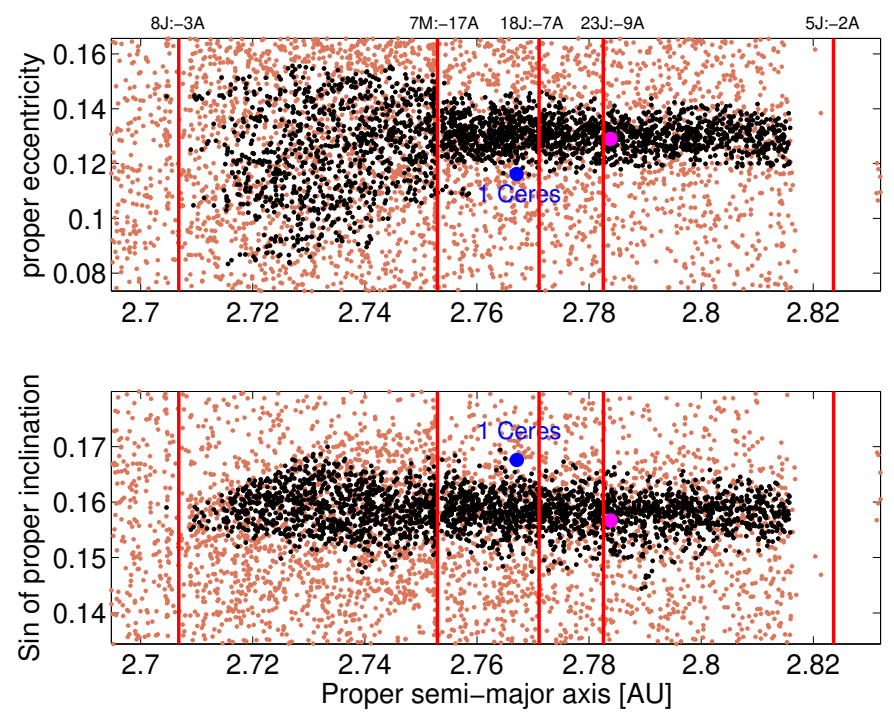

Fig. 2. Top panel: projection in the $(a, e)$ domain of 5523 AstDyS asteroids in the region of the Gefion family (orange) and of 2547 members of the Gefion HCM cluster (black). Bottom panel: projection in the $(a, \sin i)$ domain of the same asteroids. Other symbols are the same as in Fig. 1.

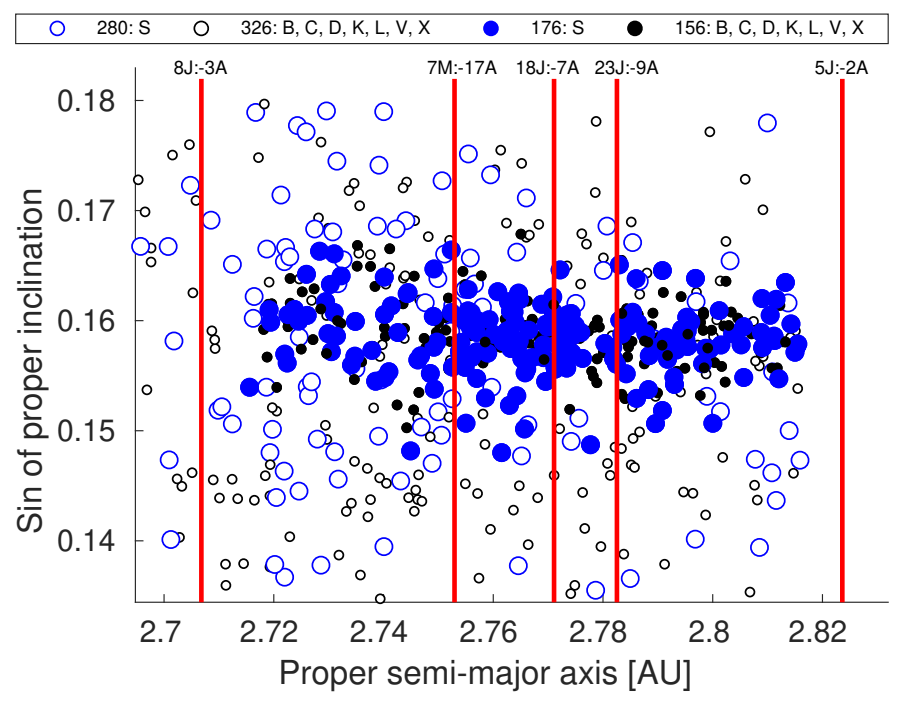

Fig. 3. An $(a, \sin i)$ projection of the 606 asteroids in the Gefion region with taxonomic information from the SDSS-MOC4 database. The inset figure legend indicates the meaning of the symbols. Open circles indicate asteroids in the background, while filled circles correspond to asteroids of the Gefion family.

the background, we used the method of DeMeo \& Carry (2013) to identify about $46.2 \%$ as S-type, $20 \%$ as L-type, $13.3 \%$ as K-type, $8.9 \%$ as X-type, $8.4 \%$ as C-type, and $3.2 \%$ as a mix of A-, B-, D-, Q-, and V-types. We show our results in Fig. 3. The S-complex objects can clearly be seen to dominate the local background, but with a significant mixing with other types of objects. Therefore, in the rest of this work, we consider the A-, B-, D-, Q-, and V-type objects as possible taxonomical interlopers and eliminate them from our family.

The Wide-field Infrared Survey Explorer (WISE) and nearEarth object WISE ${ }^{4}$ (Masiero et al. 2011; Mainzer et al. 2016)

\footnotetext{
4 ftp://wise2.ipac.cal tech.edu/staff/bauer/NEOWISE_ pass $1 /$
} 


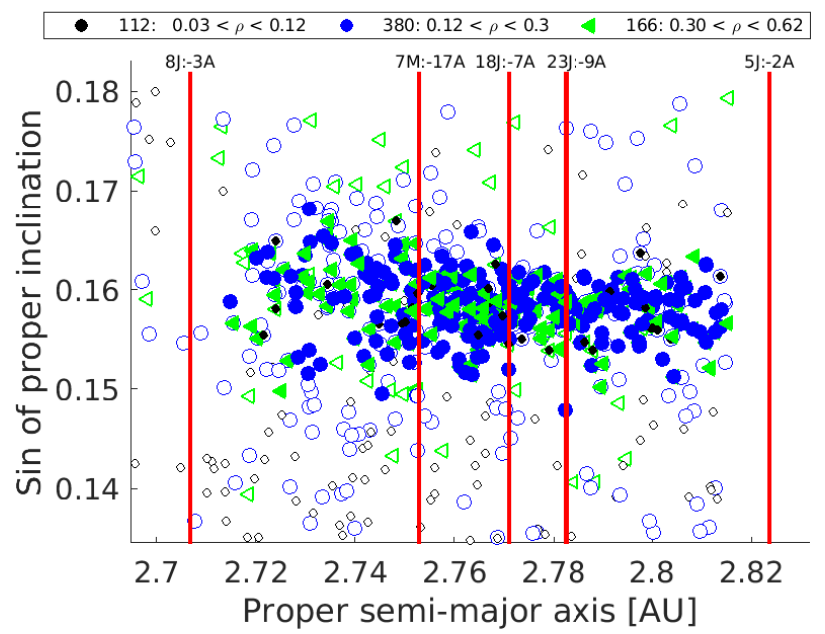

Fig. 4. An $(a, \sin i)$ projection of the 658 bodies in the Gefion region with albedo information from the WISE dataset. The inset figure legend provides the meaning of the symbols.

were also used in this work to revise asteroid diameters and albedos in the Gefion region. We identified 896 asteroids with geometric albedo ranging between $0.0278 \pm 0.0104$ and $0.6200 \pm$ 0.1510 . We found 658 of them without taxonomic information. About $57.8 \%$ of those last albedos are possibly associated with an S-type (they vary between 0.12 and 0.30 with a standard deviation of 0.05). The mean albedo found in the HCM group is 0.2609 and the median one is 0.2598 with an associated standard deviation of 0.17 (Fig. 4).

Considering a simple model with a spherical shape and a uniform density varying according to each class (DeMeo \& Carry 2013), we find that the effective diameters $(D)$ of the members in the HCM group vary between 0.82 and $71.46 \mathrm{~km}$. The total mass of the family is estimated to be $0.791 \pm 0.54 \times 10^{18} \mathrm{~kg}$. Assuming that the objects on the left-hand side of the family are lost in the 5J:-2A MMR, our family probably originates from the breakup of an S-type asteroid with a diameter $D \approx 128 \mathrm{~km}$, which is inside the allowed diameter range of $100-150 \mathrm{~km}$ mentioned in Nesvorný et al. (2009).

\section{Dynamical map}

We started our numerical exploration on the Gefion family by constructing dynamical maps of synthetic proper elements for 19000 massless particles in the $(a, \sin i)$ plane and for 18360 particles in the $(a, e)$ plane. These numbers are chosen to cover an equally spaced grid in each plan. We vary the initial semi-major axis in the interval [2.69463, 2.832] with a step size of $0.0017 \mathrm{au}$. In the $(a, e)$ plane, we increase the initial values of $e$ from 0 to 0.18 with a step of 0.0015 , while the initial values of the other elements are fixed to those of (1272) Gefion at the modified Julian date of 57200, which corresponds to June 27, 2015, and are available in AstDyS database. We use the Swift package (Levison \& Duncan 1994) to integrate the particles over $20 \mathrm{Myr}$ with a time step of 20 days. This seems to be enough to determine the stability of our test particles in the region. We included the influence of all planets and 1 Ceres. Synthetic proper elements and frequencies are calculated using the approach of Carruba (2010).

Figure 5 shows the dynamical map for particles in the background of the Gefion family (gray dots). The proper elements of real objects in the Gefion HCM dynamical group are also
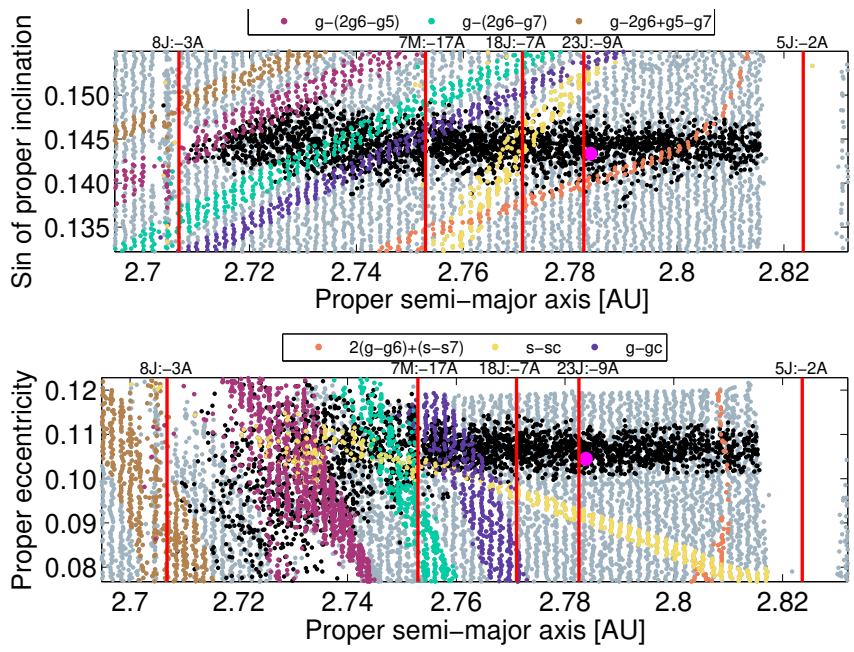

Fig. 5. Proper semi-major axis vs. sin of proper inclination (top panel) and proper eccentricity distribution (bottom panel) for objects in the region of the Gefion family (gray dots). Black dots display the proper elements of real objects in the Gefion HCM dynamical group. The inset figure legend provides the meaning of the symbols.

plotted over the dynamical map as black full dots. Vertical alignments in the maps are associated with mean-motion resonances. The particles on the right side of the family have suffered a severe depletion due to interactions with the 5J:-2A MMR. The most notable g-type resonances in the area are: the $g-\left(2 g_{6}-\right.$ $\left.g_{5}\right), g-\left(2 g_{6}-g_{7}\right)$ and $g-2 g_{6}+g_{5}-g_{7}$ resonances involving frequencies from Uranus, Saturn, and Jupiter. The first two resonances may significantly affect the determination of proper elements according to Milani \& Knezevic (1994). The resonance $2\left(g-g_{6}\right)+\left(s-s_{7}\right)$ of the $(2 g+s)$-type is also observed in the region. We notice that some asteroids have synthetic proper frequencies $\left(s\right.$ and $g$ ) very close to that of Ceres $\left(s_{c}=-59.170034\right.$, $\left.g_{c}=54.070272 \operatorname{arcsec} \mathrm{yr}^{-1}\right)$; the long-term effects of the local resonances is further studied in Sect. 5.

\section{Age determination}

To obtain a preliminary estimate of the age of the Gefion family, we calculate the isolines of maximum displacement in $a$ caused by the diurnal Yarkovsky effect (Vokrouhlický 1998, 1999). The thermal properties, such as thermal inertia or conductivity are not available. Most of the Gefion family members are asteroids of a few kilometres in diameter (only $0.94 \%$ are bigger than $10 \mathrm{~km}$ in diameter). Therefore, according to Delbo et al. (2015), higher values of the thermal conductivity $(K)$ and lower densities are required compared to the typical parameters of S-type bodies listed in Brož et al. (2013). We choose to work with $K=0.002 \mathrm{~W} \mathrm{~m}^{-1} \mathrm{~K}^{-1}$ and $\rho_{\text {bulk }}=2000 \mathrm{~kg} \mathrm{~m}^{-3}$ (associated thermal inertia of $50 \pm 4 \mathrm{~J} \mathrm{~m}^{-2} \mathrm{~s}^{-0.5} \mathrm{~K}^{-1}$, assuming that the specific heat capacities are in the range $500-680 \mathrm{~J} \mathrm{~kg}^{-1} \mathrm{~K}^{-1}$; Britt et al. 2002; Farinella et al. 1998). Our results are displayed in Fig. 6. The isolines were computed both for $T=1103 \mathrm{Myr}$ (Spoto et al. 2015) and $T=1500$ Myr. We classified only eight asteroids as dynamical interlopers.

In addition, we perform Monte Carlo simulations in which fictitious Gefion families are generated for different values of the ejection velocity parameter $\left(V_{\mathrm{EJ}}\right)$ at the current location of (1272) Gefion. First, we obtain, for each family, a histogram of the parametric target function $C$ as defined in Vokrouhlický et al. $(2006 a, b, c)$. The histogram of this function for the actual 


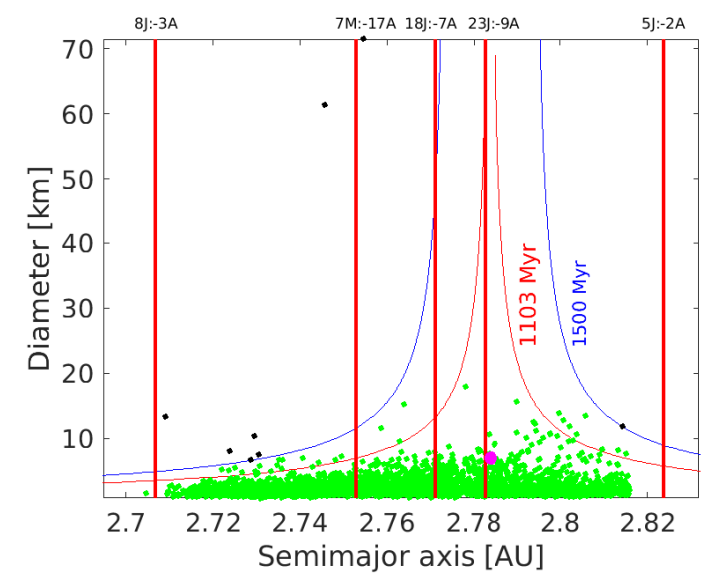

Fig. 6. Time-scale isolines of the Yarkovsky effect for the Gefion family with respect to the current location of (1272) Gefion itself. The red and blue lines correspond to $T=1103$ and $1500 \mathrm{Myr}$, respectively. The eight asteroids classified as dynamical interlopers are represented by black dots.

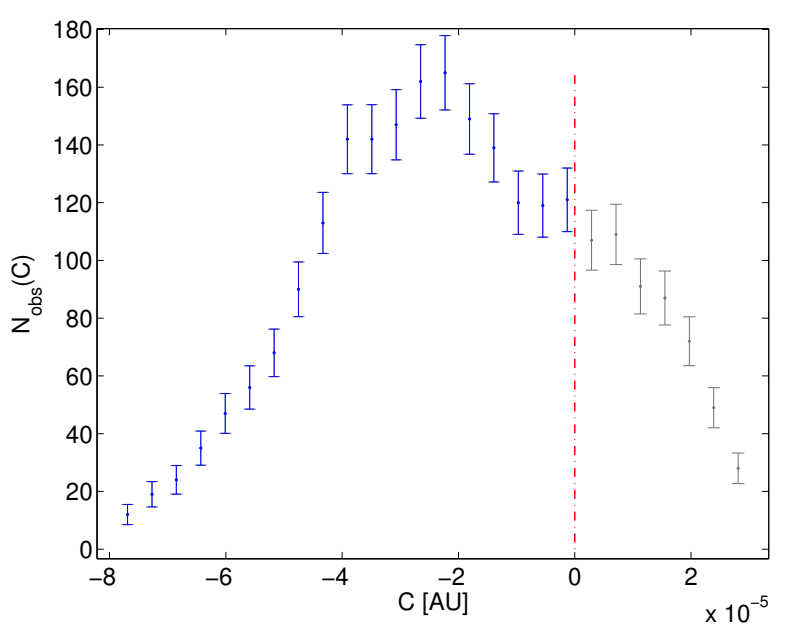

Fig. 7. Histogram of values of the $\mathrm{C}$ target function for the Gefion family.

Gefion family is presented in Fig. 7. On this occasion, 26 intervals were used starting at $C_{\min }=-7.9 \times 10^{-5}$ with a step of $\Delta C=0.42 \times 10^{-5}$ au.

The escape velocity $\left(V_{\text {esc }}\right)$ from a parent body of $128 \mathrm{~km}$ in diameter and a bulk density of $2.0 \mathrm{~g} \mathrm{~cm}^{-3}$ is estimated as $61.079 \mathrm{~m} \mathrm{~s}^{-1}$. Carruba (2016) showed that the $V_{\mathrm{EJ}}$ of the most families in the main belt did not exceed $1.5 V_{\text {esc }}$. Therefore, the maximum value of $V_{\mathrm{EJ}}$ considered here is roughly $80 \mathrm{~m} \mathrm{~s}^{-1}$. Under the influence of Yarkovsky and YORP forces, we simulated the behavior of fictitious families generated by the approach of Vokrouhlický et al. (2006b), producing distributions of the $C$-target function for different values of $V_{\mathrm{EJ}}$. The simulated C-distributions can be compared to the observed one by minimizing the $\chi^{2}$-like function of the form

$\psi_{\Delta C}=\frac{\left(N(C)-N_{\mathrm{obs}}(C)\right)^{2}}{N_{\mathrm{obs}}(C)}$,

where $N(C)$ and $N_{\text {obs }}(C)$ are the numbers of simulated and observed objects in the appropriate C-bin, respectively. The degrees of freedom for the $\chi^{2}$-like function are given by the number of intervals in $\mathrm{C}$ minus the number of parameters

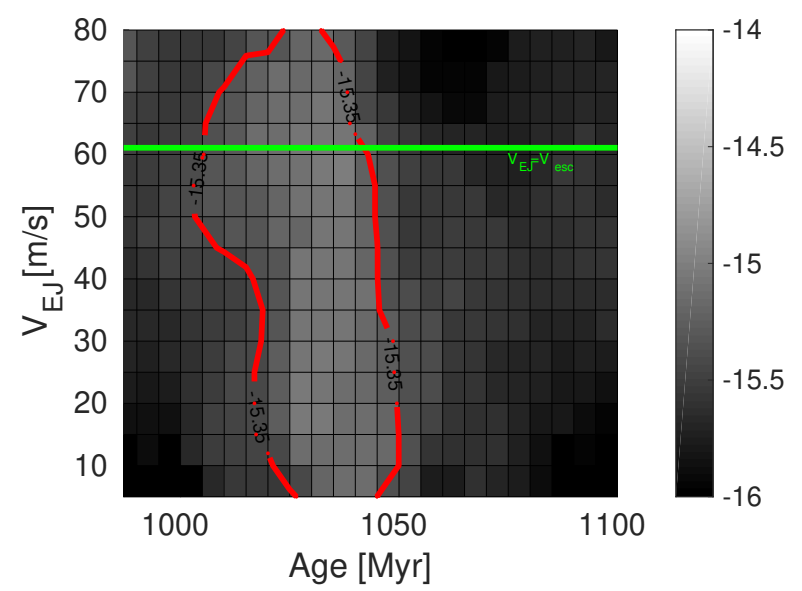

Fig. 8. Values of the $\psi \Delta C$ function in the (Age, $V$ ) plane. The red line corresponds to $\Psi \Delta C=15.2$, and the green line shows the value of the ejection velocity from (1272) Gefion.

estimated from the distribution. Our analysis was limited to a two-dimensional parameter space (age, $V_{\mathrm{EJ}}$ ). We neglect the positive $C$ values (gray lines), focusing on the left side of the family. Therefore, our distribution has 17 degrees of freedom.

In Fig. 8 we present the age and the ejection velocity parameter that produced the best fit to the observed data. The limit corresponding to $\Psi \Delta C=15.2$ yields a probability of $68.27 \%$ compatible both with the simulated and the observed distributions. We find an age of $1030_{-20}^{+15} \mathrm{Myr}$ and an ejection velocity parameter of $V_{\mathrm{EJ}}=20_{-15}^{+55} \mathrm{~m} \mathrm{~s}^{-1}$. We highlight the fact that this age estimation is compatible with the age found in Spoto et al. (2015). We further note that this uncertainty on the age is due to the method we used. However, the physical properties will affect the albedo and bulk density chosen for our Monte Carlo simulations. We re-performed the Monte Carlo simulations considering the minimum and maximum albedo for each test particle in our family, and subsequently calculated the medium age. We found almost the same age with larger uncertainty $1030_{-67}^{+19}$ Myr.

\section{Dynamical evolution of the Gefion family}

The SWIFT_RMVSY code from the Swift package (Levison \& Duncan 1994) and the SYSYCE (Swift + Yarkovsky + Stochastic Yorp + Close encounters) integrator of Carruba et al. (2015) are used in this work. We analyze the dynamical evolution of 2160 massless particles (with absolute magnitudes $H<15.03$ ) over $1.5 \mathrm{Gyr}$, in a fictitious Gefion family generated with the ejection parameter $V_{\mathrm{EJ}}$ found in Sect. 4 considering that about half of the family was lost in the 5J:-2A MMR. In our integrations, we consider the diurnal and seasonal versions of the Yarkovsky effect, described by Vokrouhlický (1998) and Vokrouhlický \& Farinella (1999), using the values of the Yarkovsky parameters typical of S-type asteroids discussed in the previous section. Two spin-axis obliquities forming angles of $0^{\circ}$ and $180^{\circ}$ with the orbital angular momentum are used in the SWIFT_RMVSY integration in order to investigate the maximum orbital mobility caused by the Yarkovsky effect, while the SYSYCE integrator accounted also for the stochastic YORP effect, as described in Bottke et al. (2015). Initial obliquity $(\epsilon)$ and angular velocity of rotation $(\omega)$ were assigned to each test asteroid. The initial orientation of the spin axis was randomly generated in the space. Changes in the two rotation-state parameters $(\omega, \epsilon)$ caused by the 


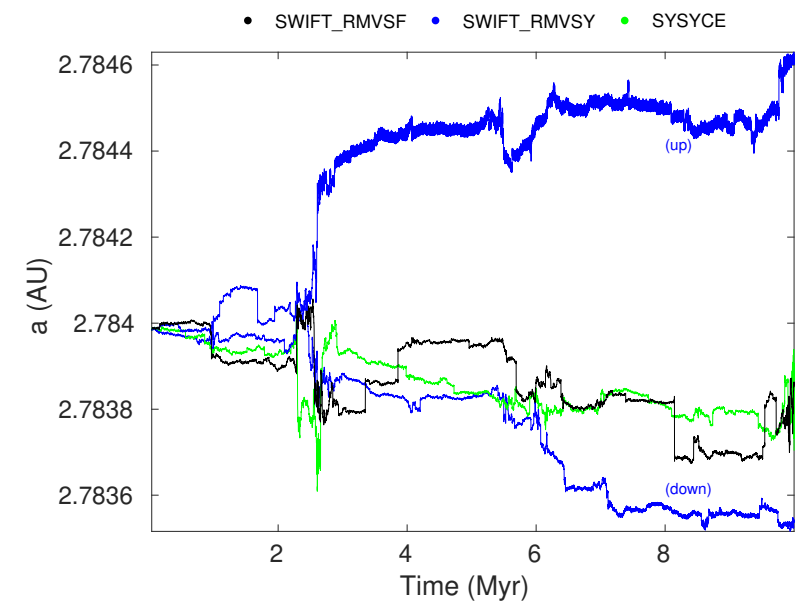

Fig. 9. Time evolution of the osculating semimajor axis of a particle in the region of Gefion using the SWIFT_RMVSY (blu line) and the SYSYCE integration (green line).

YORP effect are computed using two functions ( $f$ - and $g$-) originally derived in Čapek \& Vokrouhlický (2004) and displayed in Fig. 23 in Bottke et al. (2015). These functions are chosen to be in the regions of variance of the results of this last study. However, they were randomly changed in this zone every $\tau_{\text {YORP }}=1 \mathrm{Myr}$ in the stochastic YORP model. This makes the SYSYCE model much more costly in computation time than the SWIFT_RMVSY model. The YORP strength parameter $C_{\text {YORP }}$ was introduced in Vokrouhlický et al. (2006b) in order to adjust the uncertainties in modeling the YORP effect by multiplying this parameter by the YORP influence on $\omega$ and $\epsilon$. Here, $\left(C_{\text {YORP }}\right)$ is assumed to be 0.7. Also, in order to control the asymmetry of the YORP effect in the rotation rate for small asteroids Bottke et al. (2015) introduced the $\delta_{\text {YORP }}$ parameter, which was assumed to be 0.4 and is adopted here. Moreover, we use a typical value of 20 days for the step size of the orbits of main-belt asteroids.

As a first step, we consider an orbit in the region integrated over $10 \mathrm{Myr}$ and perturbed by (i) only the eight planets and 1 Ceres, calculated by the SWIFT_RMVSF integrator, (ii) point (i) + Yarkovsky effect (no YORP force) calculated by the SWIFT_RMVSY integrator, and (iii) point (ii) + YORP effects calculated by the SYSYCE integrator. The results of (i), (ii), and (iii) are presented in black, blue, and green lines in Fig. 9, respectively. We recall that the initial spin obliquity was fixed to $0^{\circ}$ (up) and $180^{\circ}$ (down) for the second simulation to maximize the strength of the Yarkovsky force, while it is random in the third case. As we can see, our test particle evolves towards larger values of $a$, with the SWIFT_RMVSY integrator fixing the initial spin obliquity to $0^{\circ}$. The maximum effect of the Yarkovsky force considerably changes the semi-major axis of the particle after only 3 Myr. Including the YORP effect, this oscillation is controlled and there is a stabilization in the semimajor axis. However, we want to check if this difference affects the global age estimation of our family.

Using the approach described in Carruba (2010), we calculate the proper elements of each particle. We first apply the method presented in Carruba \& Nesvorný (2016), checking the time behavior of the $\gamma_{2}\left(v_{W}\right)$ parameter, which is the kurtosis of the component of the ejection velocity field $\left(v_{W}\right)$ perpendicular to the orbital plane. In Fig. 10, we present our results for simulations both with the SWIFT_RMVSY and SYSYCE integrators. The value of $\gamma_{2}\left(v_{W}\right)$ of the simulated Gefion family reaches the value of the current Gefion Family, estimated as
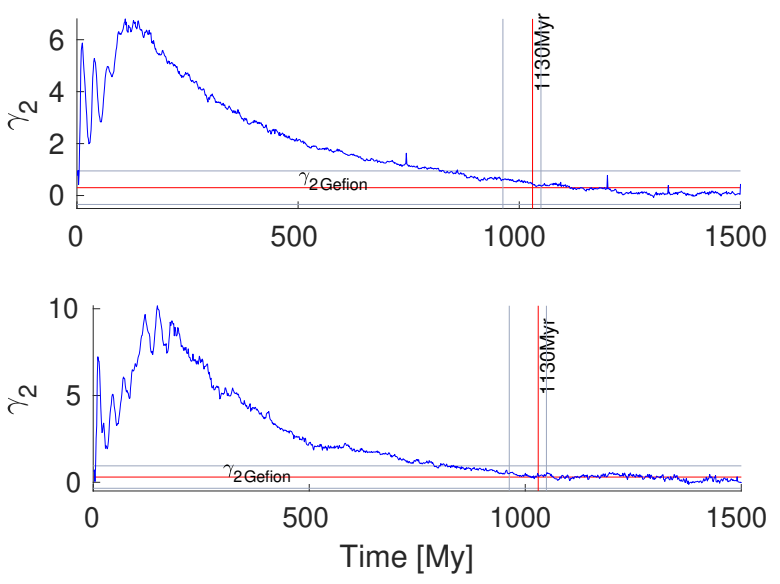

Fig. 10. Behavior of the kurtosis of the $v_{W}$ component of the ejection velocity field, using the SWIFT_RMVSY (top panel) and the SYSYCE integration (bottom panel). Vertical dashed lines display the estimated age of the family.
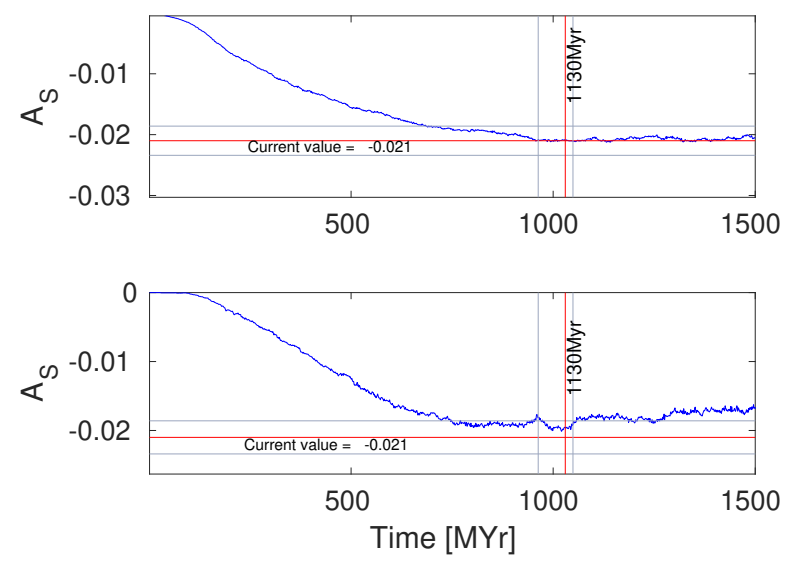

Fig. 11. Behavior of the asymmetry coefficient of the target function, with SWIFT_RMVSY (top panel) and SYSCE. The horizontal lines display the current value of $A_{S}$ and its error. Other symbols are the same as in Fig. 10.

$0.3016 \pm 0.6459$ and presented as horizontal lines in the figure, on a timescale compatible with our estimation in the previous section of the family age (vertical lines).

In order to confirm our estimation, we also apply the method presented in Aljbaae et al. (2017a,b), analyzing the degree of asymmetry of the current Gefion family. The time behavior of the asymmetry coefficient $\left(A_{\mathrm{S}}\right)$ for simulations with the SWIFT_RMVSY and SYSYCE integrators are both presented in Fig. 11. In our case, the current value of $A_{\mathrm{S}}$ is $-0.021 \pm 0.0024$. Remarkably, we find a very good agreement with the age estimated from the SWIFT_RMVSY integrator. The SYSYCE integrator leads to a trough inside the allowed age range, that could be related to a numerical artifact in our integrator.

During the $1500 \mathrm{Myr}$ of our simulation, we find that 140 asteroids ( $6.5 \%$ of our total test particles) reach orbits similar to particles in the NEOs space. The first one is detected $204 \mathrm{Myr}$ after the Gefion parent body breakup. Only four particles stay in the NEOs space for more than 5 Myr. From the 140 particles, we classify $73 \%$ as Amors with orbits exterior to the orbit of the Earth but interior to the orbit of Mars. The remaining particles are classified as Apollos; these cross the orbit of the Earth with a semimajor axes larger than that of the Earth. We also find that 
about $0.5 \%$ of the Gefion family reach the Mars-crossing space 527 Myr after the breakup.

\section{Conclusion}

In this work, we carry out a study of the Gefion asteroid family, which is a group of S-type asteroids ranging between the 8J:-3A and 5J:-2A MMRs. The 5J:-2A resonance seems to be responsible for the absence of the right side of the V-shape of this family. The Gefion region is analyzed in both dynamical and physical aspects. We show that the Gefion family probably originates from a catastrophic breakup of an S-complex-type asteroid with $D \approx 128 \mathrm{~km}$. For taxonomical reasons, we eliminated all the possible taxonomical interlopers from the family listed in Nesvorný et al. (2015). Using the method of Yarkovsky isolines we also eliminated the possible dynamical interlopers in the family including the asteroid (93) Minerva. We use the kurtosis of the $v_{W}$ component of the ejection velocity field and the $\mathrm{C}$ distribution of a fictitious Gefion family to obtain an estimation of the age of $1030_{-67}^{+19} \mathrm{Myr}$, in good agreement with previous estimates of the family age obtained by Spoto et al. (2015) or Nesvorný et al. (2005). During the entire period of our simulation, we find that $6.5 \%$ of asteroids from the Gefion family can reach the NEOs space. We also find that $73 \%$ of the Gefion family asteroids possibly belong to the Amors category and that the remaining ones can probably be classified as Apollos. We find that only $0.5 \%$ of the particles from the Gefion family reach the Mars-crossing space $527 \mathrm{Myr}$ after the breakup.

Acknowledgements. The authors are grateful to the reviewer of this article, Dr Federica Spoto, for comments and suggestions which greatly improved the quality of this work. We are indebted to Dr. Valerio Carruba for allowing us to use the multiprocessing computing system in FEG-UNESP campus of Guaratinguetá. This paper was written while the first author finished his first postdoctorate at FEG-UNESP awarded by FAPESP, Grant 13/15357-1 and started another one at the National Institute for Space Research (INPE) in Sao Jose dos Campos, Brazil, awarded by CNPq, Grant 153683/2018-0. We are grateful to the financial support from the Coordination for the Improvement of Higher Education Personnel (CAPES).

\section{References}

Aljbaae, S., Carruba, V., Masiero, J. R., Domingos, R. C., \& Huaman, M. 2017a, MNRAS, 467, 1016
Aljbaae, S., Carruba, V., Masiero, J. R., Domingos, R. C., \& Huaman, M. 2017b, MNRAS, 471, 4820

Bendjoya, P., \& Zappalà, V. 2002, Asteroids III, eds. W. F. Bottke, Jr. A. Cellino, P. Paolicchi, \& R. P. Binzel (Tucson, AZ: University Press of Arizona), 613 Bottke, W. F., Vokrouhlický, D., Walsh, K. J., et al. 2015, Icarus, 247, 191

Britt, D. T., Yeomans, D., Housen, K., \& Consolmagno, G. 2002, Asteroids III, eds. W. F. Bottke, Jr. A. Cellino, P. Paolicchi, \& R. P. Binzel (Tucson, AZ: University Press of Arizona), 485

Brož, M., Morbidelli, A., Bottke, W. F., et al. 2013, A\&A, 551, A117

Bus, S. J., \& Binzel, R. P. 2002a, Icarus, 158, 146

Bus, S. J., \& Binzel, R. P. 2002b, Icarus, 158, 106

Čapek, D., \& Vokrouhlický, D. 2004, Icarus, 172, 526

Carruba, V. 2010, MNRAS, 408, 580

Carruba, V. 2016, MNRAS, 461, 1605

Carruba, V., \& Nesvorný, D. 2016, MNRAS, 457, 1332

Carruba, V., Burns, J. A., Bottke, W., \& Nesvorný, D. 2003, Icarus, 162, 308

Carruba, V., Nesvorný, D., Aljbaae, S., \& Huaman, M. E. 2015, MNRAS, 451, 244

Cellino, A., Bus, S. J., Doressoundiram, A., \& Lazzaro, D. 2002, Asteroids III, eds. W. F. Bottke, Jr. A. Cellino, P. Paolicchi, \& R. P. Binzel (Tucson, AZ: University Press of Arizona), 633

Delbo, M., Mueller, M., Emery, J. P., Rozitis, B., \& Capria, M. T. 2015, Asteroids IV, ed. P. Michel, F. E. DeMeo, \& W. F. Bottke (Tucson, AZ: University of Arizona Press), 107

DeMeo, F. E., \& Carry, B. 2013, Icarus, 226, 723

Farinella, P., Vokrouhlický, D., \& Hartmann, W. K. 1998, Icarus, 132, 378

Ivezić, Ž., Tabachnik, S., Rafikov, R., et al. 2001, AJ, 122, 2749

Le Corre, L., Reddy, V., Bottke, W. F., Dykhuis, M., \& Nathues, A. 2015, Lunar Planet. Sci. Conf., 46, 1431

Levison, H. F., \& Duncan, M. J. 1994, Icarus, 108, 18

Mainzer, A., Bauer, J., Grav, T., et al. 2016, VizieR Online Data Catalog: $\mathrm{J} / \mathrm{ApJ} / 784 / 110$

Masiero, J. R., Mainzer, A. K., Grav, T., et al. 2011, ApJ, 741, 68

Milani, A., \& Knezevic, Z. 1994, Icarus, 107, 219

Milani, A., Cellino, A., Knežević, Z., et al. 2014, Icarus, 239, 46

Nesvorný, D., Jedicke, R., Whiteley, R. J., \& Ivezić, Ž. 2005, Icarus, 173, 132

Nesvorný, D., Vokrouhlický, D., Morbidelli, A., \& Bottke, W. F. 2009, Icarus, 200, 698

Nesvorný, D., Brož, M., \& Carruba, V. 2015, Asteroids IV, eds. P. Michel, F. E. DeMeo, \& W. F. Bottke (Tucson, AZ: University of Arizona Press), 297

Spoto, F., Milani, A., \& Knežević Z. 2015, Icarus, 257, 275

Vokrouhlický, D. 1998, A\&A, 335, 1093

Vokrouhlický, D. 1999, A\&A, 344, 362

Vokrouhlický, D., \& Farinella, P. 1999, AJ, 118, 3049

Vokrouhlický, D., Brož, M., Bottke, W. F., Nesvorný, D., \& Morbidelli, A. 2006a, Icarus, 183, 349

Vokrouhlický, D., Brož, M., Bottke, W. F., Nesvorný, D., \& Morbidelli, A. 2006b, Icarus, 182, 118

Vokrouhlický, D., Brož, M., Morbidelli, A., et al. 2006c, Icarus, 182, 92

Zappala, V., Cellino, A., Farinella, P., \& Milani, A. 1994, AJ, 107, 772 